\title{
Role of Multislice CT in the Evaluation of Non-Vascular Complications Following Hepatic Transplantation from Adult Living Donors
}

\author{
HAZEM H. SOLIMAN, M.D.* and MEDHAT MADBOULI, M.D.** \\ The Department of Radiology, Faculty of Medicine, Cairo University* and Theodor Bilharz Research Institute, Cairo**, Egypt
}

\begin{abstract}
Background: Hepatic transplantation especially from living donors has allowed widespread utilization of the procedure in management of terminal hepatic disease.

Complications following hepatic transplantation are encountered frequently. These complications may be either vascular or non-vascular complications. The non-vascular complications may include, biliary complications, infection and tumoral recurrence.

Different diagnostic radiological procedures are used for identification of these complications including ultrasound, Doppler, computed tomography and magnetic resonance imaging.

Recent advances in multislice computed tomography including the multiplanar and angiographic capabilities have expanded the role of computed tomography in the identification and differentiation of such complications.
\end{abstract}

Aim of Study: The aim of this study is to highlight the importance of multi-slice CT in the identification and evaluation of the various non-vascular post-operative complications in recipients following hepatic transplantation.

Patients and Methods: This is a prospective study that included 45 patients presenting with suspected post hepatic transplantation complications. Patients were referred to the Kasr Al-Aini Hospital Radiology Department between October 2017 to December 2019 to perform Multislice CT (MSCT) for further evaluation of underlying complications.

Results: Non-vascular complications were found in 21 cases $(46.6 \%)$. Biliary complications represented $57.1 \%$ of the non-vascular complications ( 7 cases biloma and 5 cases biliary stricture). Parenchymal complication included 2 cases of hepatic abscess $(9.5 \%)$ and 2 cases of graft rejection $(9.5 \%)$. Neoplastic complications included 4 cases recurrent HCC (19\%) and 1 case neoplastic lympho-proliferative disorder $(4.7 \%)$.

Conclusion: MSCT of the liver is a feasible non-invasive procedure for evaluating non-vascular complications following liver transplantation allowing prompt and proper intervention.

Correspondence to: Dr. Hazem H. Soliman, The Department of Radiology, Faculty of Medicine, Cairo University, Egypt
Key Words: MSCT - Hepatic - Transplantation - Complications.

\section{Introduction}

HEPATIC transplantation is the only definitive curative option for End Stage Liver Disease (ESLD) that offers cure rather than palliation. In the early 1980 s, there were several major advances such as the introduction of cyclosporine based immunosuppression, the progress in donor surgery and organ preservation that led to greatly improved results allowing liver transplantation to expand and receive universal acceptance [1]

Immunosuppressive drugs may conceal the complications following hepatic transplantation. Therefore accurate diagnostic imaging is required to aid in the delineation of such complications [2]

Complications following hepatic transplantation can be divided into vascular and non-vascular complications. The latter includes; biliary complications, recurring malignancy and infection [3].

Biliary complications are a major cause of graft dysfunction especially in children. The majority of these complications become apparent during the first 3 months. However, late biliary complication such as strictures and stones development may take months or years to develop [4].

A variety of post transplant collections including bilomas, abscesses, hematomas and localized ascites are also encountered in post hepatic transplant recepients [5].

Early detection and confirmation of graft rejection permit faster and more appropriate clinical intervention [6]. 
Many imaging modalities were used in the assessment of the post-hepatic transplantation complications including conventional angiography and Doppler studies for the assessment of vascular complications as well as MRI and MRCP for the assessment of biliary complication and ultrasonography for the assessment of the biliary complications and the post-operative collections [2].

The multiplanar capabilities of Multi-Detector row CT (MDCT) as well as its spatial and temporal resolution superiority and minimal-invasiveness and facilitated detection of hepatic anatomy, pathology and complications following liver transplantation. This is best appreciated via utilization of different post-processing reformatting techniques including Multi-Planar Reformat (MPR), Maximum Intensity Projection (MIP), and Volume Rendering (VR) [6].

MDCT is also superior to other diagnostic imaging procedures in detecting non-vascular biliary, parenchymal and neoplastic complications [6].

The aim of this study is to highlight the importance of multi-slice CT in the delineation and evaluation of the various non-vascular complications following hepatic transplantation.

\section{Patients and Methods}

\section{Study population and design:}

This is a prospective study performed between October 2017 to December 2019, on 45 adult recipients (32 males and 13 females) who performed adult-adult IIving Donor Liver Transplantation (LDLT). The age ranged between 42 years to 68 years (mean age 53.5 years). The patients were referred from the Transplantation Unit to the Radiology Department at Cairo University Hospital, Egypt. The study was approved by the Ethical Committee of our hospital.

\section{Inclusion criteria:}

- Adult patients following living donor liver transplantation with symptoms and signs suggestive of underlying post-operative complications.

- Patients in which routine ultrasonography examinations failed to accurately detect post transplantation complications and needed further MSCT evaluation.

\section{Exclusion criteria:}

- Allergy to iodinated contrast medium.

- Serum creatinine level more than $1.6 \mathrm{mg} / \mathrm{dl}$.

\section{Technique of MSCT:}

All the 45 patients have been subjected to MSCT of the abdomen and MSCTA of the liver adopting the following technique:

- Patients lied supine on the gauntry table with their arms positioned above their heads.

- A sixty-four multislice CT scanner (TOSHIBA Medical Systems, Aquillion, Japan) was used to perform the examination in all the patients.

- An $18 \mathrm{G}$ catheter was placed into a superficial antecubital vein.

- A scannogram was acquired from the dome of the right diaphragmatic copula till $20 \mathrm{~cm}$ caudally.

- Pre-contrast and triphasic post contrast sequences were obtained.

- The triphasic study was performed by computerassisted bolus-tracking technology. The Region of Interest (ROI) was placed in the abdominal aorta at the level of the celiac artery with a threshold triggered at $120 \mathrm{HU}$.

- 70-100ml of low osmolar non-ionic contrast medium (Ultravist 300) was injected at a rate of $5 \mathrm{ml} / \mathrm{sec}$.

- CT scanning parameters were; nominal section thickness $(1.25 \mathrm{~mm})$, gantry rotation speed $(0.4$ $\mathrm{sec})$, voltage $(120 \mathrm{KV})$, and current $(250 \mathrm{~mA})$.

- Patients were requested to hold their breath during the each phase of the study. However, this condition has not always been feasible as many of the patients were in a clinical condition that did not allow such act.

\section{Interpretation and data analysis:}

- Reconstruction was performed using a postprocessing workstation (Vitrea version 3.0.1, Vital Images, Plymouth, MN, USA) with slice width of $0.6 \mathrm{~mm}$ and an interval of $0.3 \mathrm{~mm}$. Different reconstructed reformat images were obtained including Multi-Planar Reformation (MPR), Maximum Intensity Projection (MIP) and Volume Rendering (VR) techniques.

- Our study focused on the non-vascular complications including; biliary complications (biliary stricture/bilomas), parenchymal complications (hepatic abscess and graft rejection), neoplastic complications (Hepatocellular Carcinoma (HCC) recurrence and lymphoproliferative disorders).

\section{Results}

- This study enrolled 45 post hepatic transplantation complicated patients ( 32 males and 13 females) 
with age ranging from 42 years to 68 years (mean age 53.5 years). HCV was the cause of liver cirrhosis in all cases.

- 21 out of the 45 cases that came to the Radiology Department from the Transplantation Unit revealed non-vascular complications Chart (1).

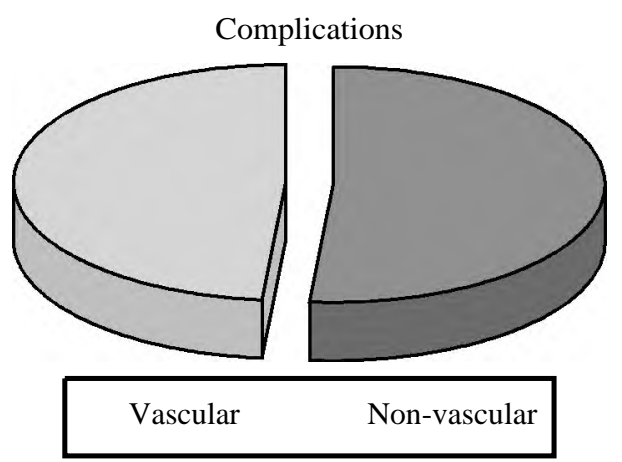

Chart (1): Percentage of non-vascular complications.

Classification of the post transplantation nonvascular complications is demonstrated in (Table $1)$.

Table (1): Number and percentage of patients according to type of non-vascular complication.

\begin{tabular}{lcc}
\hline Type of complication & $\begin{array}{c}\text { Number } \\
\text { of cases }\end{array}$ & Percentage \\
\hline Biliary: & 12 & $57.1 \%$ \\
Biloma & 7 & $33.3 \%$ \\
Biliary stricture & 5 & $23.8 \%$ \\
Parenchymal: & 4 & $19.0 \%$ \\
Hepatic abscess & 2 & $9.5 \%$ \\
Graft rejection & 2 & $9.5 \%$ \\
Neoplastic: & 5 & $23.8 \%$ \\
Recurrent HCC & 4 & $19.0 \%$ \\
Lympho-proliferative disorder & 1 & $4.7 \%$ \\
\hline
\end{tabular}

I- Biliary complications: In our study 12 out of 45 cases presented with biliary complications Chart (2). All cases were sent within the first year after transplantation.

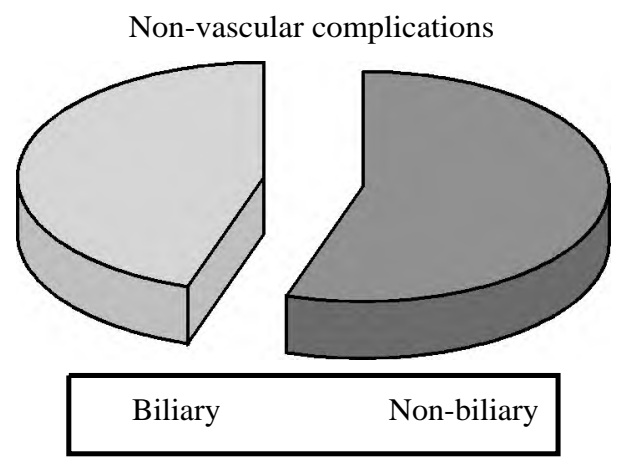

Chart (2): Percentage of biliary complications.
The complications included bilomas and biliary strictures. These complications were demonstrated by ultra-sonography examinations however, MSCT was requested for confirmation and detection of other associated complications.

A- Biliary stricture: Five out of the 12 cases sent revealed have intra-hepatic biliary radicles dilatation Fig. (1). MRCP examinations were performed for these cases which revealed the presence of peri-anastomotic biliary stricture all these cases. Biliary stents were successfully applied in the three of these cases with subsidence of the biliary obstruction.

B-Bilomas: Seven out of 12 cases sent revealed the presence of bilomas Fig. (2). MSCT examination was requested to assess the size, extent and relations of these bilomas, to enable proper selection of the best good axis of drainage. Stents were placed for drainage of these collections with successful drainage of 5 of them while the other 2 failed to resolve with further deterioration.

\section{II- Parenchymal complications:}

A- Hepatic abscess: In this study 2 cases presented with hepatic abscesses. MSCT was requested for confirmation and for assessment of the most favorable rout for drainage. The 2 abscesses were successfully drained and heavy antibiotic therapy were applied for them.

B- Graft rejection: In this study 2 cases were suspected by MSCT to represent acute graft rejection. They presented non-homogenous liver attenuation and periportal collar sign Fig. (3). Both cases were confirmed to e rejection by biopsy.

\section{III- Neoplastic complications:}

A- Recurrence of HCC: In our study 4 cases presented with hepato-cellular carcinoma recurrence in the transplanted liver after 5, 6, 8 and 10 months post-transplantation Fig. (4). No attempt for re-transplantation was performed. Of these 4 cases, 3 of them died during the first year post transplantation.

B- Lympho -proliferative disorders: In this study only one case presented with non-Hodgkin lymphoma showing diffuse bowel affection one year post-transplantation. No attempt for retransplantation was performed, and the patient died 10 months after transplantation. 

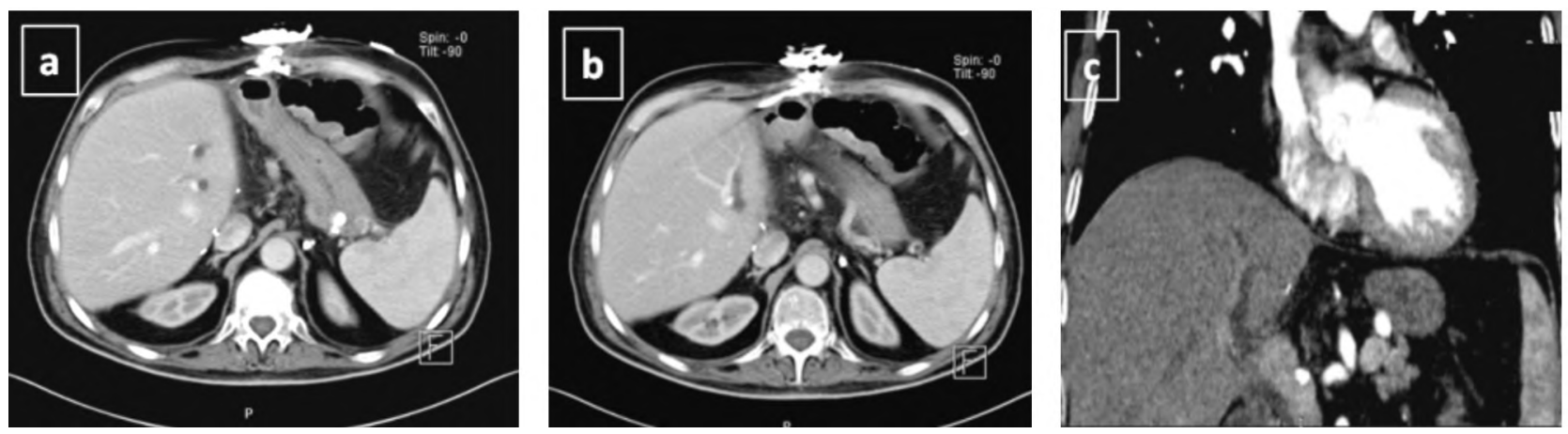

Fig. (1): 61 year old female complaining of jaundice and elevated bilirubin after hepatic transplantation. (A \& B) CT axial images show dilated intrahepatic biliary ducts more at the anterior segment of the graft (C) Coronal CT image showing common hepatic duct dilatation down to portahepatis suggesting underlying anastomotic biliary stricture.
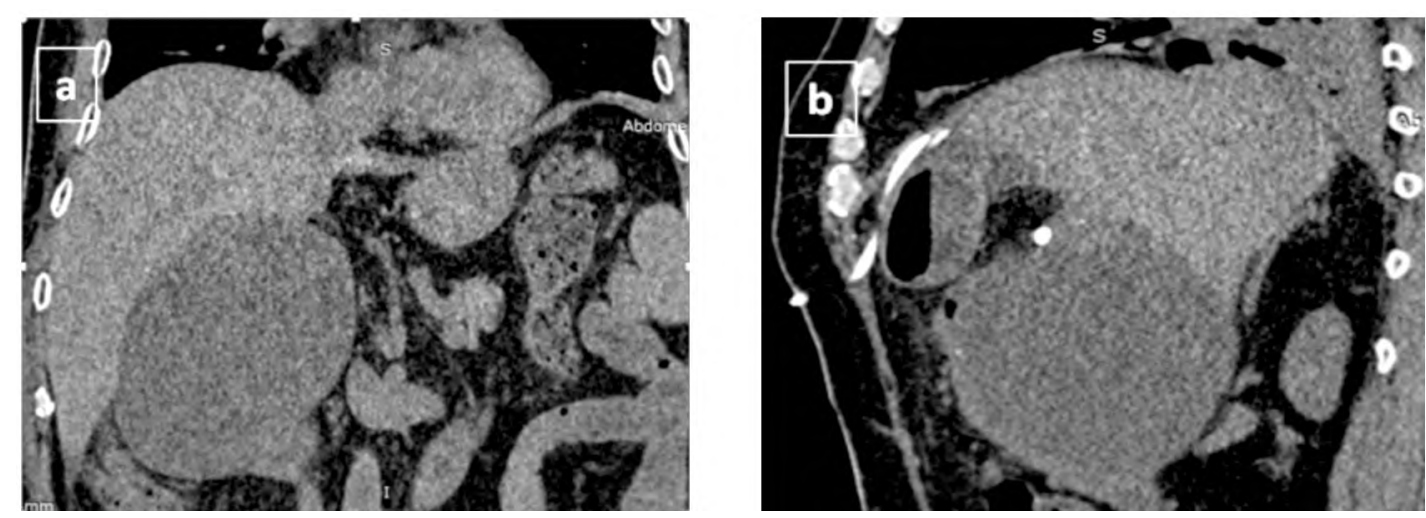

Fig. (2): 54 year old male complaining of persistent abdominal pain and fever after hepatic transplantation. (A) Coronal CT image a large subhepatic biloma (B) Sagittal CT image showing a drainiage pig tail catheter introduced into the biloma via and anterior abdominal approach.

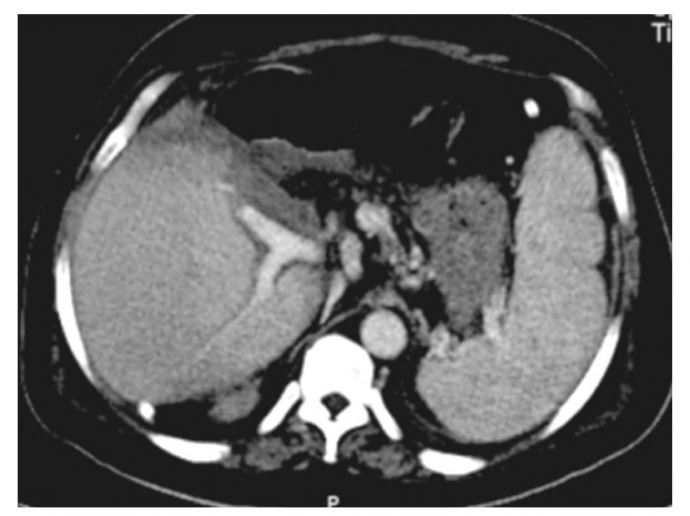

Fig. (3): 66 year old male complaining of elevate liver enzymes and right hypochondrial pain after hepatic transplantation. CT axial images showing heterogenous liver attenuation with periportal collar sign at the hepatic hilum. Graft rejection was confirmed by biopsy.
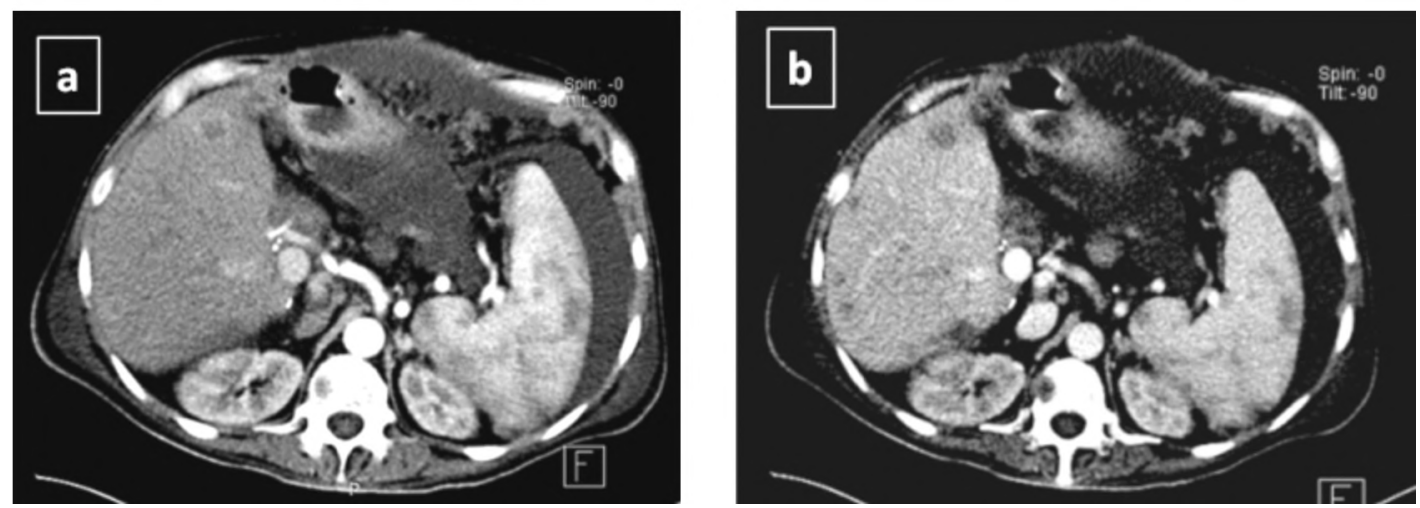

Fig. (4): 59 year old male complaining of vague abdominal pain after hepatic transplantation. (A) Axial arterial and (B) Axial portal CT images revealed multiple arterially enhancing focal lesions that display washout in the portal phase consistent with recurrent multicentric HCC. In addition there is moderate abdominal ascites. 


\section{Discussion}

Radiology has undoubtedly contributed to the development and success of liver transplantation because of the role it plays in the in recognition and management of complications. LDLT requires intensive radiological evaluation; each imaging modality provides a range of information and more than one modality is often needed to obtain the pre-surgical information and to detect intra or postoperative complications [4]

Ultrasound is utilized as the first step in the evaluation of the hepatic transplantation donors and recipients. However, CT and MRI are considered the superior modalities in delineation of the hepatic vascular, parenchymal and biliary anatomy in both hepatic donors and recipients [7].

Yeon et al., [6] reported that MDCT is superior to catheter angiography as $\mathrm{CT}$ is a noninvasive procedure. MDCT also has advantages over ultrasound being non-operator dependent and not affected by the patient's body habitus. MDCT is also more accurate in demonstrating non-vascular complications, such as, bile duct necrosis, bile leaks, biliary stricture, neoplastic recurrence and abscesses.

Singh et al., [4] stated that biliary complications are the second most common complications sent for MSCT examination confirmation to detect the need for further ERCP or MRCP assessment.

Quiroga et al., [8] pointed out that biliary complications arise in approximately $13-19 \%$ of recipients after hepatic transplantation. They included bile leaks, anastomotic strictures and sludge formation.

Biliary complications were found to represent about $57.1 \%$ of cases of our study, and that was because most of cases referred for MSCT were targeted for detection of biliary complications. These cases were diagnosed by US study, but further assessment was needed to detect the extent of bilomas, as retro-gastric ones may be missed by US, as well as to confirm biliary leak and to detect axis of drainage or any developed hepatic abscess or infection in the biliary collection.

Boraschi et al., [9] reported that biliary strictures are either anastomotic or non anastomotic. The anastomotic strictures may result from procedural difficulties during the transplantation or as a result of post biliary leakage scarring and fibrosis.
In this study 12 cases were found to have strictures or biliary leak at the anastomotic site, confirmed by further ERCP or MRCP studies.

Singh et al., [4] showed that bilomas can present as either parenchymal hypodense lesions on CT or as extra-parenchymal perihepatic or peritoneal collections.

Bilomas were seen in 7 of our patients $(33.3 \%$ of the cases). Although they were were detected by ultrasound, MSCT was required for confirmation and delineation of the route of drainage as previously explained.

Malignancy is well recognized as a potential complication of long-term immune-suppression. Immune-compromised patients have a much higher incidence of malignancy in comparison to the general population [9].

Scarsbrook et al., [10] reported that non-Hodgkin lymphoma and squamous skin carcinoma are the most common malignancies seen in LDLT in addition to recurrence of HCC. Immuno-compromised post hepatic transplantation patients are at higher risk for post-transplantation lymphoproliferative disease.

In our study only one case had post transplanation pathologically proven non-Hodgkin lymphoma with 4 cases of recurrent HCC.

We had two cases that presented with hepatic abscesses. MSCT was requested for confirmation and for demonstration of the best route for drainage.

Our study had few limitations; our sample size was limited to certain complications, as only patients with vascular and to a much less extent biliary complications were refereed for MSCT and MSCTA. This limited our capability to highlight the value of MSCT and MSCTA in the assessment of other varieties of post-transplantation complications. Furthermore it made the rate of nonvascular complications in our results slightly higher than what was encountered in the previously reported studies.

\section{Conclusion:}

With the advanced techniques of MSCT, our study showed that MSCT of the liver is a noninvasive method for delineating post hepatic transplantation non-vascular complications giving a chance for proper and swift intervention for the urgent cases. 


\section{References}

1- ABDELAZIZ O., SAMIE A.A. and METWALLY L.I.: Multi-slice computed tomography imaging of the post transplant complications in the recipients after living donor liver transplantation. The Egyptian Journal of Radiology and Nuclear Medicine, Dec. 1, 44 (4): 697 704, 2013.

2- ABDEL-RAHMAN H.M., ENABA M.M., HASAN D.I., TANTAWY F.A. and MORSY M.M.: Role of multislice $\mathrm{CT}$ and magnetic resonance cholangiography in preoperative evaluation of potential donor in living related liver transplantation. The Egyptian Journal of Radiology and Nuclear Medicine, Mar. 1, 47 (1): 33-42, 2016.

3- LEE I.S., PARK S.H., CHOI S.J., SHIM Y., AHN S.J., KIM K.W., KIM K.K., JEONG Y.M. and CHOE Y.H.: Diagnostic Performance of Multidetector Computerized Tomography in the Detection of Abdominal Complications Early and Late After Liver Transplantation: A 10-Year Experience. InTransplantation proceedings, Dec. 1 (Vol. 50, No. 10, pp. 3673-80), 2018.

4- MIRAGLIA R., MARUZZELLI L., CARUSO S., MARRONE G., CAROLLO V., SPADA M., LUCA A. and GRIDELLI B.: Interventional radiology procedures in pediatric patients with complications after liver transplantation. Radiographics, Mar., 29 (2): 567-84, 2009.

5- SINGH A.K., NACHIAPPAN A.C., VERMA H.A., UPPOT R.N., BLAKE M.A., SAINI S. and BOLAND G.W.:
Post-operative imaging in liver transplantation: What radiologists should know. Radiographics, Mar., 30 (2): 339-51, 2010

6- FEDERLE M.P. and KAPOOR V.: Complications of liver transplantation: Imaging and intervention. Radiologic Clinics, Nov. 1, 41 (6): 1289-305, 2003.

7- KIM S.Y., KIM K.W., KIM M.J., SHIN Y.M., LEE M.G. and LEE S.G.: Multidetector row CT of various hepatic artery complications after living donor liver transplantation. Abdominal imaging, Sep. 1, 32 (5): 635-43, 2007.

8- MOSTAFA I., ABD-EL AAL M., SYAM M., REFAY R., EL METENI M., HAMZA A., FATHY M., ABD-EL AAL A., SAFAAN H., EL MONAYERI M. and EL DORRY A.: HCV recurrence in adult living related liver transplantation (LRLT): Initial results of a starting program in Egypt. Liver Transplantation, 11: 456-62, 2005.

9- QUIROGA S., SEBASTIA M.C., MARGARIT C., et al.: Complications of orthotopic liver transplantation: Spectrum of findings with helical CT. Radiographics, 21 1085-9, 2001.

10- BORASCHI P. and DONATI F.: Complications of orthotopic liver transplantation: Imaging findings. Abdominal imaging, Mar. 1, 29 (2): 189-202, 2004.

11- SCARSBROOK A.F., WARAKAULLE D.R., DATTANI M. and TRAILL Z.: Post-transplantation lymphoproliferative disorder: The spectrum of imaging appearances. Clinical radiology, Jan. 1, 60 (1): 47-55, 2005.

\section{دور الآثمة المقطعية متعددة الشرائح فى تقييم المضاعفات غير الوعائية

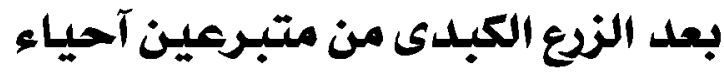

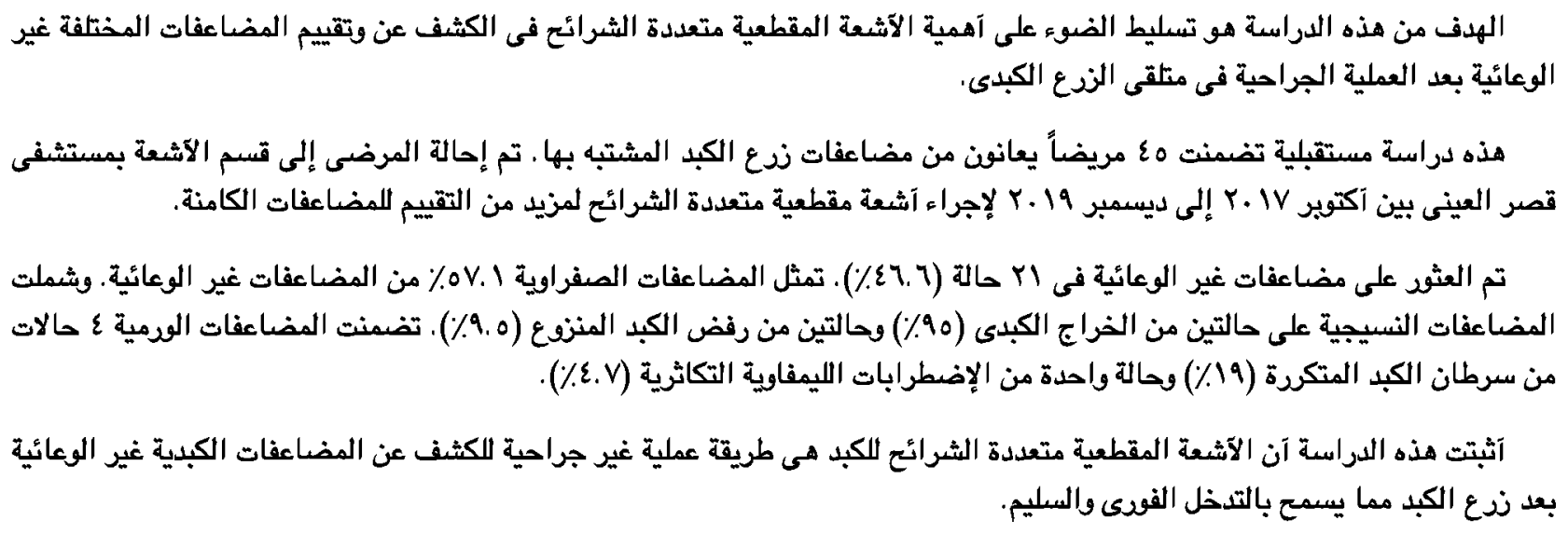

